\title{
Conjugators of Fuchsian groups and quasiplatonic surfaces
}

\author{
Ernesto Girondo and Jürgen Wolfart
}

\begin{abstract}
Let $G$ be a Fuchsian group containing two torsion free subgroups defining isomorphic Riemann surfaces. Then these surface subgroups $K$ and $\alpha K \alpha^{-1}$ are conjugate in $\mathbb{P S L}(2, \mathbb{R})$, but in general the conjugating element $\alpha$ cannot be taken in $G$ or a finite index Fuchsian extension of $G$. We will show that in the case of a normal inclusion in a triangle group $G$ these $\alpha$ can be chosen in some triangle group extending $G$. It turns out that the method leading to this result allows also to answer the question how many different regular dessins of the same type can exist on a given quasiplatonic Riemann surface.
\end{abstract}

\section{Introduction}

Quasiplatonic Riemann surfaces of genus $g>1$ can be characterized by the fact that their surface groups $K$ are normal subgroups of triangle groups $\Delta$. It is well known that for every genus there are only finitely many isomorphism classes of quasiplatonic surfaces, and that two such surfaces are isomorphic if and only if their surface groups $K, K^{\prime}$ are conjugate in $\mathbb{P S L}(2, \mathbb{R})$. All questions concerning classification and Galois actions on families of quasiplatonic surfaces lead therefore to problems of the following type: Let $K$ be a finite index subgroup of a Fuchsian group $G$. Given $\alpha \in \mathbb{P S L}(2, \mathbb{R})$ such that $\alpha K \alpha^{-1}$ is another subgroup of $G$, we cannot expect $\alpha$ to be an element of $G$ or of a finite index extension of $G$, so where can $\alpha$ come from? In this paper we will give complete answers in the case that both groups are triangle groups (Thms. 6,7 and 8) and sufficiently complete answers if at least $G$ is a triangle group (Thms. 5 and 9, Corollaries 10 and 11). This case has consequences for the construction of all regular dessins d'enfants of a given type on Riemann surfaces of genus $>1$ (Thms. 4, 12 and 13), giving also a small contribution to the wide open question how to generate all possible dessins on a given Belyi surface. The parallel question in which cases regular dessins of different types determine isomorphic Riemann surfaces has been treated recently by the first author [2] and by Singerman and Syddall [8] (there mainly restricted to types $(2, m, n)$ but also including uniform dessins).

The structure of the paper is as follows: 
In Section 2 we introduce conjugators and basic facts about them. In Section 3 we treat several interesting examples. Section 4 is devoted to triangle groups, Belyi surfaces and dessin d'enfants. The main results of the paper are contained in Section 5, where the conjugators of triangle groups inside other triangle groups are studied in detail. Some consequences for regular dessins are given in Section 6.

\section{Finite index conjugate subgroups of Fuchsian groups}

Definition 1 Let $K, G$ be two Fuchsian groups such that $K<G$. We call

$$
C(G, K):=\left\{\alpha \in \mathbb{P S L}(2, \mathbb{R}) \mid \alpha K \alpha^{-1}<G\right\}
$$

the conjugator of $K$ inside $G$.

The conjugator is not necessarily a group, since it does not behave well under composition and taking inverses. But a rather obvious feature of $C(G, K)$ is:

Lemma 1 Let $K \subset G$ be a finite index inclusion of Fuchsian groups. Then

$$
C(G, K) \supseteq N(G) N(K),
$$

where $N(\cdot)$ denotes the normalizer in $\mathbb{P S L}(2, \mathbb{R})$, and the right hand side denotes the set $\{a b \mid a \in N(G), b \in N(K)\}$.

Remark 2 Note that an equivalent definition for the conjugator of $K$ inside $G$ is

$$
C(G, K)=\left\{\alpha \in \mathbb{P S L}(2, \mathbb{R}) \mid K<\alpha^{-1} G \alpha\right\} .
$$

$C(G, K)$ consists of a finite number of residue classes of $N(K)$, due to the fact that there are finitely many subgroups of $G$ that are conjugate to $K$. It is also easy to see that the group $\langle C(G, K)\rangle$ generated by $C(G, K)$ satisfies

$$
K<G<\langle C(G, K)\rangle<\operatorname{Comm}(G),
$$

where

$\operatorname{Comm}(G):=\left\{\gamma \in \mathbb{P S L}(2, \mathbb{R}) \mid G \cap \gamma G \gamma^{-1}\right.$ has finite index in $G$ and in $\left.\gamma G \gamma^{-1}\right\}$ is the commensurator of $G$.

A famous theorem by Margulis, see [5], states that $\operatorname{Comm}(\Gamma)$ is again Fuchsian if and only if the Fuchsian group $\Gamma$ is not of arithmetic type (see [11] for a definition of arithmeticity). We find therefore, in the non arithmetic case, that the inclusion of $G$ inside $\operatorname{Comm}(G)$, and hence that of $K$ inside $\langle C(G, K)\rangle$, has finite index. This is in general not true in the arithmetic cases, for examples see the next section. 
Definition 2 Let $K, G$ be Fuchsian groups, $K<G$. We denote

$$
n_{G}(K)=\#\left\{H \mid H<G, \exists g \in \mathbb{P S L}(2, \mathbb{R}) \text { s.t. } H=g K g^{-1}<G\right\}
$$

the number of different subgroups of $G$ that are conjugate to $K$, and

$$
N_{K}(G)=\#\left\{H \mid H>K, \exists g \in \mathbb{P S L}(2, \mathbb{R}) \text { s.t. } H=g G g^{-1}>K\right\}
$$

the number of different supergroups of $K$ that are conjugate to $G$.

Note that $n_{G}(K)$ and $N_{K}(G)$ are in general independent of each other.

\section{$3 \quad$ Examples of large conjugators}

In this section we show that conjugators are in general not contained in the set $N(G) N(K)$ used in Lemma 1 and generate very large groups.

Theorem 3 There exist infinitely many arithmetic Fuchsian groups $G$, normal torsion free subgroups $K$, and $\alpha \notin N(G) N(K), \alpha \in \mathbb{P S L}(2, \mathbb{R})$, such that $K$ is also normal in $\alpha^{-1} G \alpha$. The conjugating element $\alpha$ does not belong to any Fuchsian group extending $K$.

Proof.- First let $\Gamma$ be the elliptic modular group $\mathbb{P S L}(2, \mathbb{Z})$ and $G:=\Gamma(p)$ the principal congruence subgroup of prime level $p$

$$
\Gamma(p):=\left\{\left(\begin{array}{ll}
a & b \\
c & d
\end{array}\right) \in \operatorname{SL}(2, \mathbb{Z}) \mid b \equiv c \equiv 0, a \equiv d \equiv 1 \bmod p\right\} / \pm E_{2}
$$

(where $E_{2}$ denotes the $2 \times 2$ unit matrix), $K:=\Gamma\left(p^{2}\right)$ the principal congruence subgroup of level $p^{2}$, and let $\alpha$ be given in normalized form as

$$
\alpha=\frac{1}{\sqrt{p}}\left(\begin{array}{cc}
p & 0 \\
0 & 1
\end{array}\right) \in \mathbb{P S L}(2, \mathbb{R}) .
$$

It is easy to verify that $K$ is a normal subgroup in both $G$ and

$$
\alpha^{-1} G \alpha=\left\{\left(\begin{array}{ll}
a & b \\
c & d
\end{array}\right) \in \mathrm{SL}(2, \mathbb{Z}) \mid c \equiv 0 \bmod p^{2}, a \equiv d \equiv 1 \bmod p\right\} / \pm E_{2}
$$

and that $\alpha$ is not contained in $N(G)=N(K)=\Gamma$. Since no proper power $\alpha^{n}$ of $\alpha$ has an integer trace, $\alpha$ cannot belong to a finite index extension of $K$, so the claim follows.

These considerations extend almost without change to other arithmetic Fuchsian groups. To obtain cocompact examples, consider a definite quaternion algebra $B$, for simplicity defined over $\mathbb{Q}$. Let $\Gamma$ be the norm unit group in a maximal order $\mathcal{O}$ of $B$. If $p$ is unramified in $B$, i.e. for almost all primes, $B$ embeds into $M\left(2, \mathbb{Q}_{p}\right)$ and by the strong approximation theorem, $\Gamma$ is dense in 
$\mathbb{P S L}\left(2, \mathbb{Z}_{p}\right)$, see e.g. $[6], \S 5.2$. So $\Gamma(p)$ and $\Gamma\left(p^{2}\right)$ can be defined in the same way as above and $\alpha \in B$ can be chosen as congruent to

$$
\left(\begin{array}{ll}
p & 0 \\
0 & 1
\end{array}\right) \bmod p^{2} \mathcal{O}
$$

Even if $\Gamma$ has torsion elements, the traces in $\Gamma(p)$ are congruent to $2 \bmod p$, whence $\Gamma(p)$ and $\Gamma\left(p^{2}\right)$ are torsion free.

We remark in passing that the quotient groups $G / K, \alpha^{-1} G \alpha / K$ constructed here are not isomorphic. In Section 5, Example 1, we will meet even (mostly non-arithmetic!) cases for which $K$ is a normal subgroup of $G$ but a non-normal subgroup of $\alpha^{-1} G \alpha$.

\section{Normal surface subgroups of triangle groups}

We now turn our attention to the case of the normal inclusions of a torsion free group $K$ inside a Fuchsian triangle group $G$. Any such inclusion corresponds to a regular dessin d'enfant on the surface uniformized by the surface group $K$. The study of $C(G, K)$ turns out to be a very important point in this case, and has consequences concerning dessins.

We have to recall some basics about triangle groups, dessins d'enfants and Belyi surfaces. We shall expose the main ideas without giving proofs, as the interested reader can find them easily in the literature (see, e.g. [1], [4], [13]).

\subsection{Triangle groups and Belyi surfaces}

Let $T(l, m, n)$ be a hyperbolic triangle with angles $\pi / l, \pi / m$ and $\pi / n$. Consider the group generated by the reflections in the sides of $T(l, m, n)$, and let $\Delta(l, m, n)$ be the index 2 subgroup formed by its orientation preserving elements.

Definition 3 The group $\Delta(l, m, n)$ constructed as above is a Fuchsian triangle group of type $(l, m, n)$.

If $\gamma_{0}, \gamma_{1}$ and $\gamma_{\infty}$ are the (hyperbolic) turns with angles $2 \pi / l, 2 \pi / m$ and $2 \pi / n$ around the corresponding vertices of $T(l, m, n)$, then

$$
<\gamma_{0}, \gamma_{1}, \gamma_{\infty} ; \gamma_{0}^{l}=\gamma_{1}^{m}=\gamma_{\infty}^{n}=\gamma_{\infty} \gamma_{1} \gamma_{0}=1>
$$

is a presentation of $\Delta(l, m, n)$. The union of the generating triangle $T(l, m, n)$ with one of its images under a reflection in a side forms a fundamental domain for $\Delta(l, m, n)$. Accordingly, $\Delta(l, m, n)$ corresponds to a tessellation of the hyperbolic plane $\mathbb{D}$ with triangles of angles $\pi / l, \pi / m$ and $\pi / n$.

The quotient space under the action of $\Delta(l, m, n)$ is the Riemann sphere with three marked points of order $l, m, n$ respectively. Triangle groups are rigid, in the sense that two triangle groups of the same type are conjugate in $\operatorname{PSL}(2, \mathbb{R})$, any conjugating element being an isometry of the hyperbolic plane sending one generating triangle into the other. 
Triangle groups play a very important role in several branches of mathematics. They appear very naturally in different mathematical contexts like monodromy groups of hypergeometric differential equations. They are central in the theory of Belyi surfaces and Belyi maps, giving a link connecting triangle groups with Galois Theory.

Definition 4 A covering of the Riemann sphere by a compact Riemann surface that ramifies only above three points is a Belyi function. Accordingly, the covering space is called a Belyi surface.

A very famous result by Belyi (see e.g. [4], [13]) shows that this kind of surfaces are deeply related to the field of algebraic numbers, since they correspond exactly to those Riemann surfaces which can be defined, as algebraic curves, by polynomial equations with coefficients in $\overline{\mathbb{Q}}$.

\subsection{Dessins d'Enfants}

One of the most surprising facts about the theory of Belyi surfaces is the observation, early pointed out by Grothendieck and others, that they can be studied in a quite simple combinatorial way.

If $\beta: X \longrightarrow \hat{\mathbb{C}}$ is a Belyi function, the preimage of the real interval $] 0,1[\subset \hat{\mathbb{C}}$ forms the edges of a bipartite graph $\mathcal{D}$ embedded into the compact Riemann surface $X$, such that the connected components of $X \backslash \mathcal{D}$, the so-called faces of $\mathcal{D}$, are homeomorphic to open discs. The $\beta$-preimages of 0 are the white vertices and the $\beta$-preimages of 1 are the black vertices of $\mathcal{D}$. Such kind of graphs were called by Grothendieck dessins d'enfants due to their simplicity.

The Belyi function $\beta$, hence the complex structure of $X$, is completely determined by $\mathcal{D}$ in a very simple way. And $\mathcal{D}$ is in turn completely determined by its monodromy group $\mathcal{M}$, that is a permutation group on the set of edges describing completely the incidence relations, defined as follows:

Label the edges of $\mathcal{D}$ with numbers from 1 to $N$. Each vertex of $\mathcal{D}$ has an associated cycle, given simply by the edges incident to it (arranged, say, in counterclockwise order). Since $\mathcal{D}$ is bipartite, the cycles corresponding to the vertices of one colour are pairwise disjoint. Let $\sigma_{0}$ and $\sigma_{1}$ be the product of the cycles around the white and black vertices respectively. Since $\mathcal{D}$ is connected, they generate a permutation group acting transitively on the edges. It can be seen that each cycle of $\sigma_{\infty}=\left(\sigma_{0} \sigma_{1}\right)^{-1}$ describes half the edges encountered around each of the faces. The group generated by $\sigma_{0}, \sigma_{1}$ and $\sigma_{\infty}$ is the monodromy group $\mathcal{M}$ of $\beta$.

To avoid too much case-by-case analysis we will assume in the following that our compact Riemann surfaces $X$ have genus $g>1$. Then there is an epimorphism $\theta: \Delta(l, m, n) \rightarrow \mathcal{M}$, where $l$ and $m$ are the least common multiples of the valencies of the black and white vertices respectively, and $n$ is half the least common multiple of the valencies of the faces, and we have automatically $\frac{1}{l}+\frac{1}{m}+\frac{1}{n}<1$, so $\Delta(l, m, n)$ is a Fuchsian triangle group as constructed above. Moreover, the surface $X$ is reconstructed as $\mathbb{D} / K$, where $K$ is the (not necessarily torsion free!) preimage via $\theta$ of the stabilizer of an edge. The trivial 
projection $\mathbb{D} / K \longrightarrow \mathbb{D} / \Delta(l, m, n) \cong \hat{\mathbb{C}}$ is a Belyi function, having $\mathcal{D}$ as associate dessin, and every Belyi function can be written in this way. Note in particular the well known fact that Belyi surfaces correspond exactly to quotient spaces of the hyperbolic plane $\mathbb{D}$ under the action of subgroups of triangle groups.

Definition 5 Let $\mathcal{D}$ be the dessin on the Riemann surface $X=\mathbb{D} / K$ where $K$ is a finite index subgroup of the Fuchsian triangle group $\Delta(l, m, n)$ and $\mathcal{D}$ belongs to the Belyi function

$$
\beta: \mathbb{D} / K \longrightarrow \mathbb{D} / \Delta(l, m, n) \cong \hat{\mathbb{C}}
$$

whose l.c.m. of the ramification orders above $0,1, \infty$ are $l, m, n$ respectively. Then we call $(l, m, n)$ the type of $\mathcal{D}$, and we will call $\mathcal{D}^{\prime}$ a renormalization of $\mathcal{D}$ if it belongs to a Belyi function $\delta \circ \beta$ where $\delta$ is an automorphism of $\hat{\mathbb{C}}$ permuting $0,1, \infty$.

An example of a renormalization is to replace $\beta$ by $\beta /(\beta-1)$ meaning for the dessin an interchange of black vertices and centers of the faces.

\subsection{Regular dessins and quasiplatonic surfaces}

Two classes of dessins are particularly interesting, namely uniform and regular dessins. A dessin is uniform when black vertices, white vertices, and faces have respectively constant valency. It corresponds to the inclusion of a torsion free, hence uniformizing, Fuchsian group inside a triangle group. We call a dessin regular - it will be automatically uniform - if the automorphism group acts transitively on its set of edges, an automorphism of the dessin being simply a colour-preserving bijection of the vertices compatible with relation of adjacency; it can be always seen as a restriction of a holomorphic automorphism of $X$ to the dessin. Equivalently, the surface group associated to a regular dessin is normally contained in the corresponding triangle group, and the Belyi function $\beta$ is a Galois covering.

Riemann surfaces that contain a regular dessin are called quasiplatonic. They are also called surfaces with many automorphisms, since any surface that is close enough in moduli space to a quasiplatonic one has a strictly smaller number of automorphisms.

A quasiplatonic surface may contain several regular dessins of different types (see [2]). On the other hand, suppose that $\mathcal{D}$ is a dessin embedded in the surface $X$ uniformized by $K$. Then $\mathcal{D}$ is induced by the inclusion $K \triangleleft \Delta$ for some triangle group $\Delta$. Any other regular dessin $\mathcal{D}^{\prime}$ on $X$ of the same type as $\mathcal{D}$ is given by a second inclusion $K \triangleleft \Delta^{\prime}$, where $\Delta^{\prime}=\alpha \Delta \alpha^{-1}$ for some $\alpha$. Caution: "other" means that $\mathcal{D}$ and $\mathcal{D}^{\prime}$ are not renormalizations of each other, it means $\Delta \neq \Delta^{\prime}$. Both are contained in the normalizer $N(K)$ which is again a triangle group $\tilde{\Delta}$. Therefore we have a first result on multiple quasiplatonic surfaces of a given type - more precise information will follow in Section 6. 
Theorem 4 Let $\mathcal{D}$ be a regular dessin on the quasiplatonic surface $X=\mathbb{D} / K$ induced by the normal inclusion $K \triangleleft \Delta$, where $K$ is torsion free and $\Delta$ a Fuchsian triangle group. Let $\tilde{\Delta}$ be the normalizer $N(K)$. Then all regular dessins of the same type as $\mathcal{D}$ on $X$ correspond to elements in $C(\tilde{\Delta}, \Delta)$ modulo $N(\Delta)$. Their number is bounded by $n_{\tilde{\Delta}}(\Delta)$.

We obtain even a one-to-one correspondence if $K$ is normally contained in all $\Delta^{\prime} \subset \tilde{\Delta}$ conjugate to $\Delta$. In this case, $n_{\tilde{\Delta}}(\Delta)$ is the precise number of dessins on $X$ of the same type as $\mathcal{D}$.

\subsection{Type-preserving isomorphisms between quasiplatonic surfaces}

In the consideration of Galois actions on families of quasiplatonic surfaces one is often led to the question if two normal surface subgroups $K, K^{\prime}$ of a given triangle group $\Delta$ (mostly with isomorphic quotients $\Delta / K \cong \Delta / K^{\prime}$ ) are conjugate. An easy example: for every prime $q \equiv \pm 1 \bmod 7$ there are three different normal surface subgroups $K_{i}, i=1,2,3$, of $\Delta(2,3,7)$ all with quotients isomorphic to $\mathrm{PSL}_{2}\left(\mathbf{F}_{q}\right)$ giving three non-isomorphic but Galois conjugate MacbeathHurwitz curves, see [9] and Corollary 11 below; more complicate examples can be found in [10]. The same argument as above shows that $K$ and $\alpha K \alpha^{-1}$ are both normal in $\Delta$ if and only if $K$ is normal in $\Delta$ and $\alpha^{-1} \Delta \alpha$, both triangle groups being contained in the normalizer $N(K)=\tilde{\Delta}$ of $K$. Again we have

Theorem 5 If there is an $\alpha \in \mathbb{P S L}(2, \mathbb{R})$ such that the surface groups $K$ and $\alpha K \alpha^{-1}$ are both normal subgroups of the triangle group $\Delta$, then $\alpha^{-1} \in$ $C(\tilde{\Delta}, \Delta)$, where $\tilde{\Delta}=N(K)$ is the normalizer of $K$.

\section{The conjugator of a triangle group inside an- other triangle group}

Both results about multiple dessins of the same type and about the typepreserving isomorphisms lead to the study of conjugators of triangle groups inside other triangle groups. We start by taking into account the cases $\Delta_{1}<\Delta_{2}$ such that no other triangle group lies in between $\Delta_{1}$ and $\Delta_{2}$.

D. Singerman obtained in $1972[7]$ the list of all possible inclusion relations between two triangle groups. One can easily check that there is a subset of just eight generating elements of the list, in the sense that any of the inclusions can be expressed as a concatenation of some of the generating ones. This generating 
system is

$$
\begin{gathered}
\Delta(n, n, n) \triangleleft_{3} \Delta(3,3, n) \\
\Delta(n, n, m) \triangleleft_{2} \Delta(2, n, 2 m) \\
\Delta(2, n, 2 n)<_{3} \Delta(2,3,2 n) \\
\Delta(3, n, 3 n)<_{4} \Delta(2,3,3 n) \\
\Delta(2,7,7)<<_{9} \Delta(2,3,7) \\
\Delta(3,8,8)<_{10} \Delta(2,3,8) \\
\Delta(4,4,5)<_{6} \Delta(2,4,5) \\
\Delta(3,3,7)<_{8} \Delta(2,3,7),
\end{gathered}
$$

where the subindices denote the index.

For the next result, we consider three separate cases for the second inclusion, according to the three situations $n \notin\{m, 2 m\}, n=m$, and $n=2 m$.

Theorem 6 Table 1 shows $C\left(\Delta_{2}, \Delta_{1}\right), n_{\Delta_{2}}\left(\Delta_{1}\right)$ and $N_{\Delta_{1}}\left(\Delta_{2}\right)$, where $\Delta_{1}<\Delta_{2}$ runs through the fundamental inclusions of triangle groups in the list 2.

\begin{tabular}{|c|c|c|c|c|c|}
\hline case & $\Delta_{1}$ & $\Delta_{2}$ & $C\left(\Delta_{2}, \Delta_{1}\right)$ & $n_{\Delta_{2}}\left(\Delta_{1}\right)$ & $N_{\Delta_{1}}\left(\Delta_{2}\right)$ \\
\hline i & $\Delta(n, n, n)$ & $\Delta(3,3, n)$ & $\Delta(2,3,2 n)$ & 1 & 1 \\
\hline ii a & $\Delta(n, n, m)$ & $\Delta(2, n, 2 m)$ & $\Delta(2, n, 2 m)$ & 1 & 1 \\
\hline ii b & $\Delta(m, m, m)$ & $\Delta(2, m, 2 m)$ & $\Delta(2, m, 2 m)$ & 1 & 3 \\
\hline ii c & $\Delta(m, 2 m, 2 m)$ & $\Delta(2,2 m, 2 m)$ & $\Delta(2,4,2 m)$ & 2 & 1 \\
\hline iii & $\Delta(2, n, 2 n)$ & $\Delta(2,3,2 n)$ & $\Delta(2,3,2 n)$ & 3 & 1 \\
\hline iv & $\Delta(3, n, 3 n)$ & $\Delta(2,3,3 n)$ & $\Delta(2,3,3 n)$ & 4 & 1 \\
\hline v & $\Delta(2,7,7)$ & $\Delta(2,3,7)$ & $\Delta_{2} \Delta(2,4,7)$ & 9 & 2 \\
\hline vi & $\Delta(3,8,8)$ & $\Delta(2,3,8)$ & $\Delta_{2} \Delta(2,6,8)$ & 10 & 2 \\
\hline vii & $\Delta(4,4,5)$ & $\Delta(2,4,5)$ & $\Delta_{2} \Delta(2,4,10)$ & 6 & 2 \\
\hline viii & $\Delta(3,3,7)$ & $\Delta(2,3,7)$ & $\Delta_{2} \Delta(2,3,14)$ & 8 & 2 \\
\hline
\end{tabular}

Table 1: Conjugators of the fundamental inclusions of triangle groups

Proof.- Let $\Delta_{1}<\Delta_{2}$ be any of the inclusions listed. Let $T_{1}$ and $T_{2}$ be the triangles that define $\Delta_{1}$ and $\Delta_{2}$ respectively (one half of their fundamental domains). Suppose we fix $\Delta_{1}$. If it is contained in a conjugate of $\Delta_{2}$, the vertices of $T_{1}$ must be vertices of the tessellation of the unit disc associated to $T_{2}$ of order multiple the order they had as fixed points of $\Delta_{1}$. Reciprocally, if we fix $\Delta_{2}$, any subgroup conjugate to $\Delta_{1}$ has to have the vertices of $T_{1}$ among the vertices of the tessellation given by $\Delta_{2}$, with the same observation about the ramification order as above.

This observation, applied to a case by case analysis of the inclusions, proves the result. To illustrate the procedure, let us consider the case i) in detail. Suppose that $T_{2}$ generates $\Delta_{2}$ of type $(3,3, n)$, and that $\Delta_{1}<\Delta_{2}$. A given vertex $z$ of $T_{1}$ must coincide with some order $n$ vertex of the tessellation induced by $T_{2}$. We can suppose then that $T_{2}$ has its angle $\pi / n$ vertex at $z$, which in turn determines the location of $T_{2}$ up to a turn fixing $z$, and we can suppose that an 
angle $\pi / 3$-vertex lies inside $T_{1}$. It can be easily seen that all the order $n$ vertices of the tessellation generated by $\Delta_{2}$ coincide with vertices of the tessellation generated by $\Delta_{1}$ if and only if one of the sides of $T_{1}$ bisects $T_{2}$ (see Figure 1; the black lines indicate the tessellation of the hyperbolic plane with triangles $T_{2}=T(3,3,4)$, the dotted white lines the tessellation with $\left.T_{1}=T(4,4,4)\right)$. Therefore a $(n, n, n)$ group can be included in only one $(3,3, n)$ group, hence $N_{\Delta_{1}}\left(\Delta_{2}\right)=1$. A similar argument shows that $\Delta(3,3, n)$ contains only one $\Delta(n, n, n)$, i.e. $n_{\Delta_{2}}\left(\Delta_{1}\right)=1$.

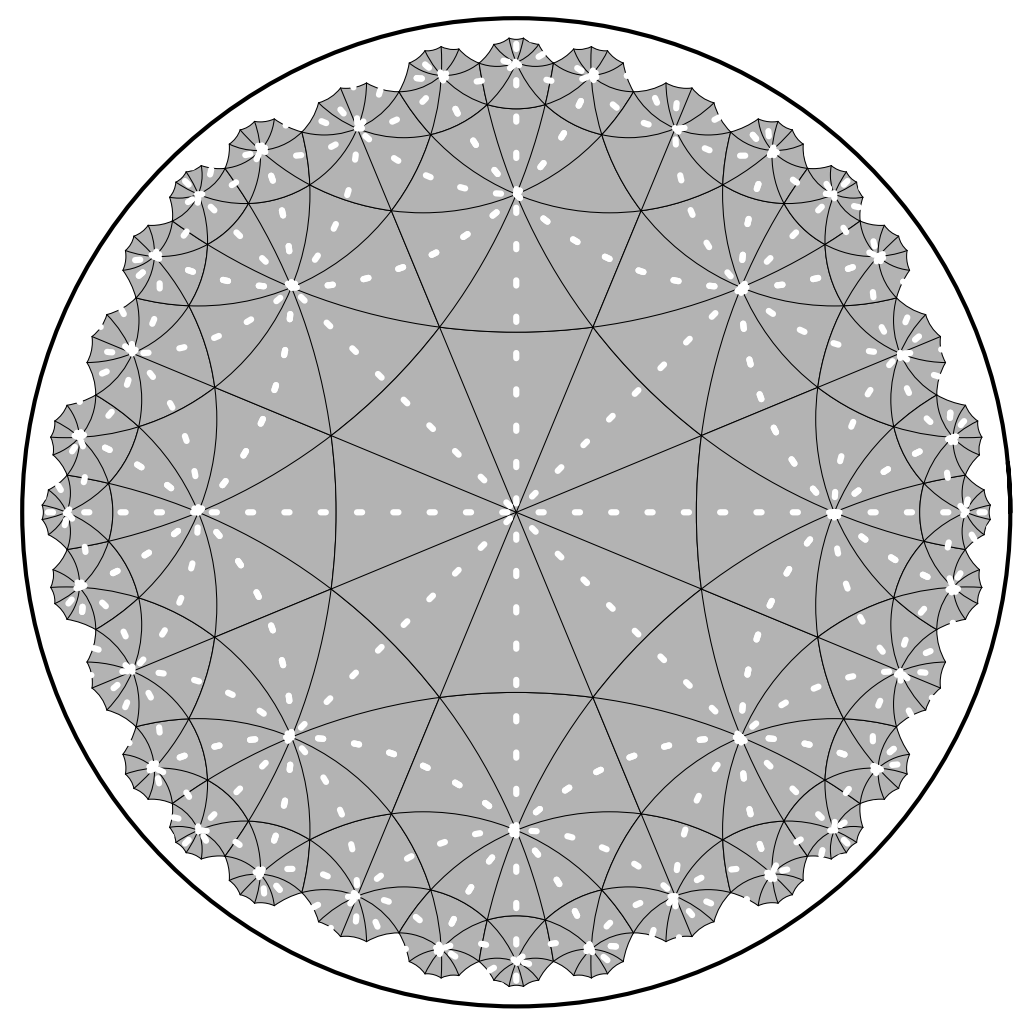

Figure 1: i) The inclusion $\Delta(n, n, n)<\Delta(3,3, n)$ in the case $n=4$.

The last four cases are more complicated. For the inclusion $\Delta_{1}=\Delta(3,8,8)<$ 
$\Delta_{2}=\Delta(2,3,8)$ of case vi), for example, we first observe that the fixed points of order 8 and of order 3 of $\Delta_{1}$ are also fixed points of $\Delta_{2}$ of the same order. Suppose both groups share the fixed point of order 3 drawn as the center of the unit disc in Figure 2. If this point is a vertex of $T_{1}$ then the other two are fixed points of order 8 . In the correct hyperbolic distance we find precisely six such fixed points of order 8 of $\Delta_{2}$, all leading to fundamental triangles for the same group $\Delta_{1}$. Therefore all possible triangle subgroups of $\Delta_{2}$ with signature $(3,8,8)$ are already uniquely determined by the choice of a fixed point of order 3. All these fixed points belong to the same $\Delta_{2}$-orbit, hence

$$
n_{\Delta_{2}}\left(\Delta_{1}\right) \leq\left(\Delta_{2}: \Delta_{1}\right)=10
$$

On the other hand, the $\Delta_{2}$-conjugates of $\Delta_{1}$ form an orbit of length precisely 10 because the normalizer $\Delta_{3}$ of $\Delta_{1}$ is the triangle group of signature $(2,6,8)$ and intersects $\Delta_{2}$ in $\Delta_{1}$, see [Ta]. This argument shows moreover

$$
C\left(\Delta_{2}, \Delta_{1}\right)=\left\{\alpha \beta \mid \alpha \in \Delta_{2}, \beta \in \Delta_{3}\right\}
$$

and

$$
N_{\Delta_{1}}\left(\Delta_{2}\right)=\left(\Delta_{3}: \Delta_{1}\right)=2
$$
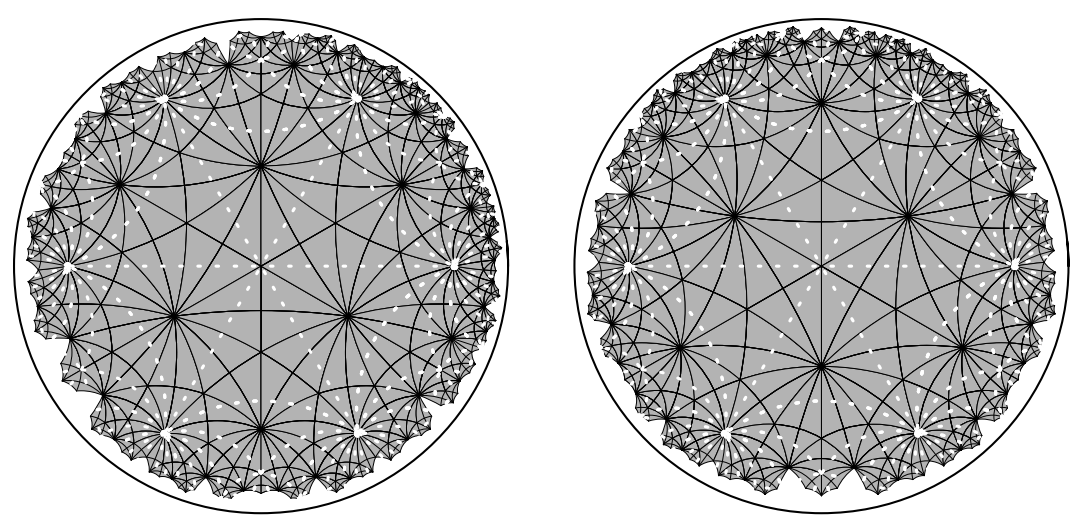

Figure 2: vi. The two $\Delta(2,3,8)$ groups containing a given $\Delta(3,8,8)$.

All other cases follow from a rather obvious analysis similar to those exposed here looking at the corresponding figures of all the inclusions involved (figures like 2 and 3 but related to the other inclusions). Due to problems of space we cannot provide so many figures here, but the interested reader may find them in the homepage [3]. 

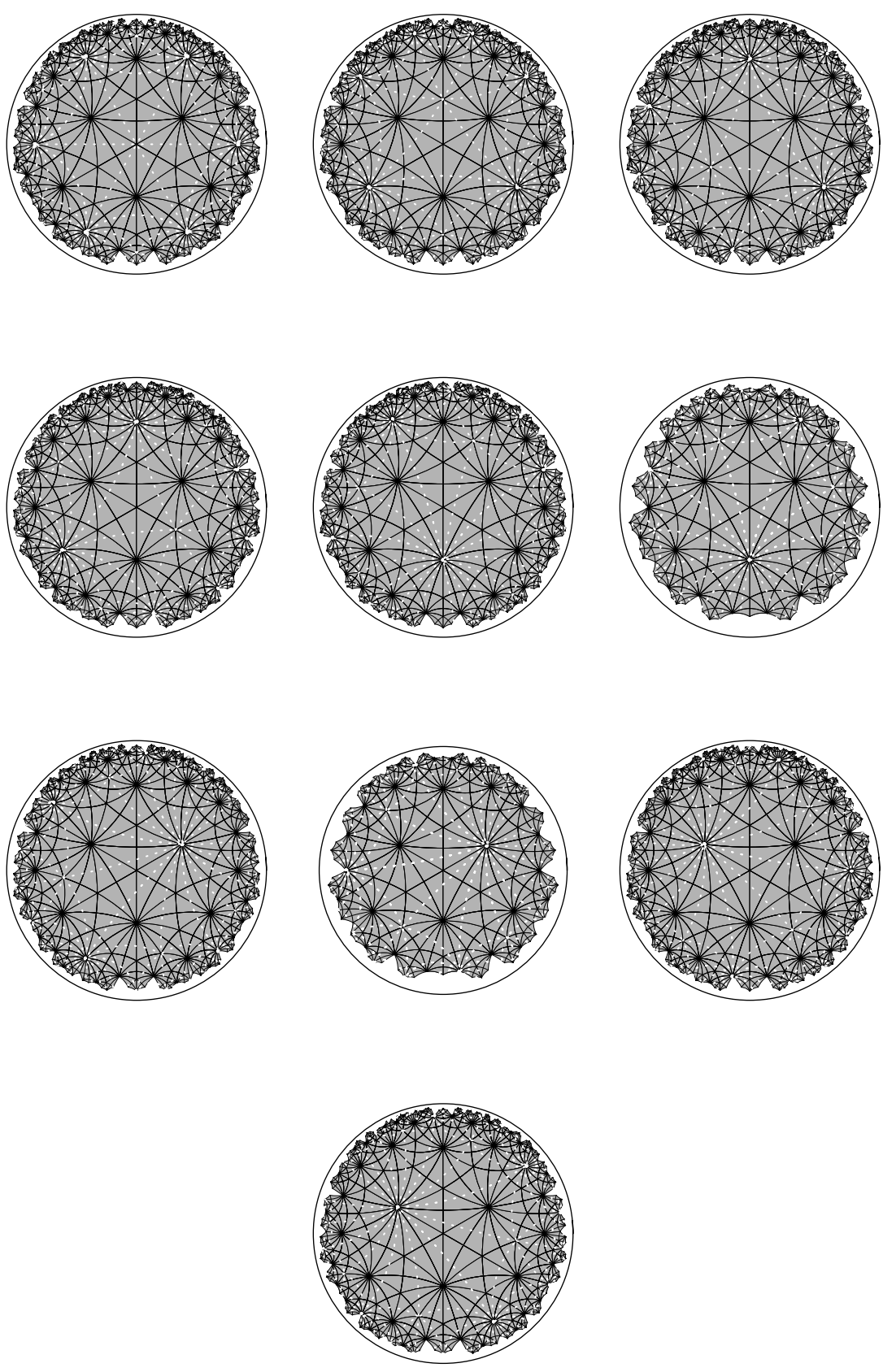

Figure 3: vi. The ten groups of type $\Delta(3,8,8)$ contained inside a given $\Delta(2,3,8)$. 
Now, if $\Delta_{1}<\Delta_{2}$ is an inclusion different to those in the list 2 , there is a third triangle group $\Delta$ lying between $\Delta_{1}$ and $\Delta_{2}$. These cases can be treated in different ways. The first possibility is to apply Theorem 6 step by step to show that $C\left(\Delta_{2}, \Delta_{1}\right)$ contains only the obvious conjugators. Here it is not a priori clear that between $\Delta_{1}$ and a conjugate $\alpha \Delta_{2} \alpha^{-1}$ of $\Delta_{2}$ there is a conjugate to $\Delta$. One can overcome this difficulty by a refinement of Singerman's methods [7] showing that between any pair of Fuchsian groups $\Delta_{1}^{\prime}<\Delta_{2}^{\prime}$ of the same signature as $\Delta_{1}$ and $\Delta_{2}$ there is an intermediate group of the same signature as $\Delta$. We will follow more closely a second possibility, i.e. using the geometric tools in the proof of the previous theorem. Only the following families of inclusions have to be considered:

$$
\Delta(n, n, n)<_{6} \Delta(2,3,2 n), n>3,
$$

with intermediate groups $\Delta(2, n, 2 n)$ or $\Delta(3,3, n)$ and

$$
\begin{aligned}
& \Delta(n, 2 n, 2 n) \triangleleft_{2} \Delta(2,2 n, 2 n) \triangleleft_{2} \Delta(2,4,2 n), n>2, \\
& \Delta(n, 4 n, 4 n) \triangleleft_{2} \Delta(2,2 n, 4 n)<_{3} \Delta(2,3,4 n), n>1 .
\end{aligned}
$$

The right hand side groups $\Delta_{2}$ are maximal triangle groups with the only exception $\Delta(2,4,8)$. Almost all groups involved are non-arithmetic, for them we know therefore by Margulis' theorem $C\left(\Delta_{2}, \Delta_{1}\right)=\Delta_{2}$, see Section 2 . Only the two-step inclusions between arithmetic triangle groups need a closer geometric look. We will explain the details in the most difficult case, the only three-step inclusion

$$
\Delta_{1}=\Delta(4,8,8) \triangleleft_{2} \Delta(2,8,8) \triangleleft_{2} \Delta(2,4,8)<_{3} \Delta(2,3,8)=\Delta_{2}
$$

(see [12], §4, case (III) with an obvious little correction). Every fixed point of $\Delta_{1}$ is an order 8 fixed point of $\Delta_{2}$, whence we can suppose that $T_{1}$ and $T_{2}$ have a common vertex of angle $\pi / 8$, without loss of generality the center 0 of the hyperbolic unit disc, see Figure 4.

If we fix $T_{2}$ (hence $\Delta_{2}$ ), $T_{1}$ (hence the subgroup $\Delta_{1}$ ) is uniquely determined by the location of its vertex of angle $2 \pi / 4$, that has to be chosen among the order 8 fixed points of $\Delta_{2}$. But all possible such vertices in the correct distance of 0 result from one of them by rotation around 0 with angles $2 \pi k / 8$. This means

$$
C\left(\Delta_{2}, \Delta_{1}\right)=\Delta_{2}, \quad N_{\Delta_{1}}\left(\Delta_{2}\right)=1
$$

and - counting the different choices of $T_{1}$ for fixed $T_{2}$ modulo the action of $\Delta_{2}$, in other words the residue classes of $\Delta_{2}$ modulo the normalizer $\Delta(2,8,8)$ of $\Delta_{1}$ - we get $n_{\Delta_{2}}\left(\Delta_{1}\right)=6$.

The other cases are easier and can be treated in the same way. For the relevant tesselations we refer to the pictures in [3]. To summarize the considerations of this section: 


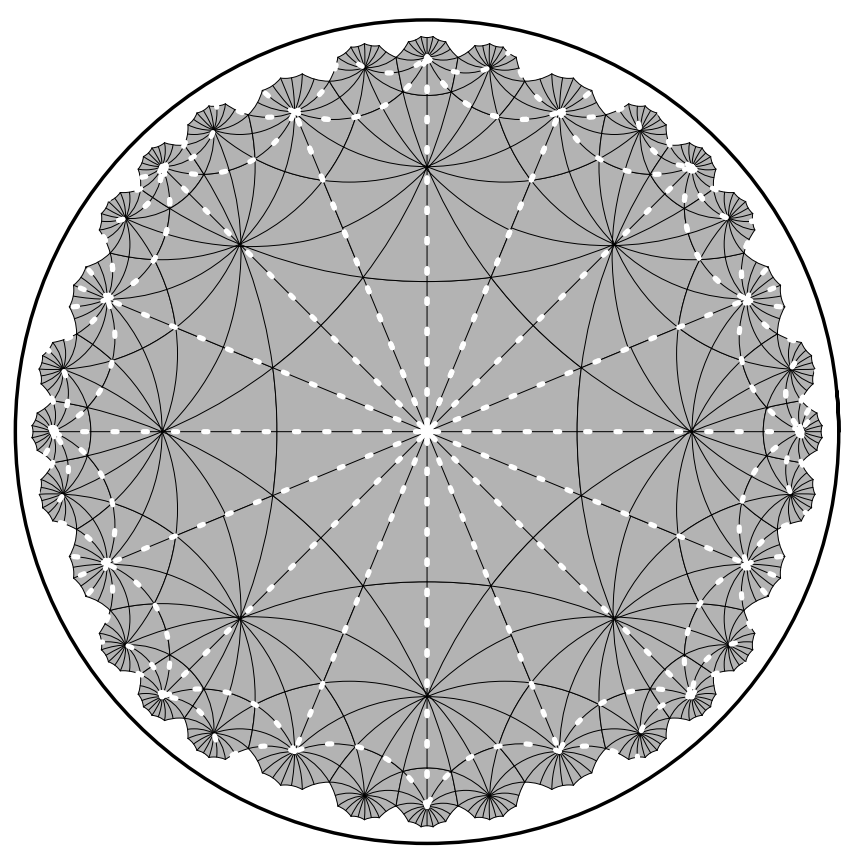

Figure 4: The three-step inclusion: $\Delta(4,8,8)<\Delta(2,3,8)$. 
Theorem 7 Let $\Delta_{1}<\Delta_{2}$ be an inclusion between two triangle groups. Then $C\left(\Delta_{2}, \Delta_{1}\right)=N\left(\Delta_{2}\right) N\left(\Delta_{1}\right)$.

And, as a consequence, we obtain:

Theorem 8 Let $\Delta_{1}$ and $\Delta_{2}$ be two triangle groups such that $\Delta_{1}<\Delta_{2}$. Then i) $n_{\Delta_{2}}\left(\Delta_{1}\right)=\left(N\left(\Delta_{2}\right): N\left(\Delta_{1}\right) \cap N\left(\Delta_{2}\right)\right)$

ii) $N_{\Delta_{1}}\left(\Delta_{2}\right)=\left(N\left(\Delta_{1}\right): N\left(\Delta_{1}\right) \cap N\left(\Delta_{2}\right)\right)$.

Proof.- Suppose that $\alpha \Delta_{1} \alpha^{-1}<\Delta_{2}$. By Theorem 7 we know that $\alpha=\gamma_{2} \gamma_{1}$ where $\gamma_{i} \in N\left(\Delta_{i}\right)$. Therefore $\alpha \Delta_{1} \alpha^{-1}=\gamma_{2} \Delta_{1} \gamma_{2}^{-1}$, so the conjugations of $\Delta_{1}$ inside $\Delta_{2}$ take part simply in $N\left(\Delta_{2}\right)$. But, clearly, if $\gamma_{2}^{-1} \tilde{\gamma}_{2} \in N\left(\Delta_{1}\right)$ then $\gamma_{2} \Delta_{1} \gamma_{2}^{-1}=\tilde{\gamma}_{2} \Delta_{1} \tilde{\gamma}_{2}^{-1}$, and we obtain i).

The proof of ii) follows by a similar argument.

Theorem 9 If the $\mathbb{P S L}(2, \mathbb{R})$-conjugate surface groups $K$ and $K^{\prime}$ are both normal subgroups of the triangle group $\Delta$, then $K^{\prime}=\alpha K \alpha^{-1}$ for some $\alpha \in N(\Delta)$ or $N(\tilde{\Delta})$ where $\tilde{\Delta}$ denotes the normalizer $N(K)$ of $K$ in $\mathbb{P S L}(2, \mathbb{R})$.

Proof.- Theorems 5 and 7 tell us $\alpha^{-1} \in C(\tilde{\Delta}, \Delta)=N(\tilde{\Delta}) N(\Delta)$, what is equivalent to $\alpha \in N(\Delta) N(\tilde{\Delta})$. Now a closer look into all possible inclusion relations between triangle groups, see [7] and [12], shows that we have $N(\Delta)<$ $N(\tilde{\Delta})$ or $N(\tilde{\Delta})=\tilde{\Delta}$. In both cases one of the factors in $N(\Delta) N(\tilde{\Delta})$ can be omitted.

As a special case we have

Corollary 10 If moreover $\Delta=N(K)$, i.e. if $\Delta / K$ is the full automorphism group of the quasiplatonic surface $X=\mathbb{D} / K$, we have $\alpha \in N(\Delta)$.

Finally we obtain a well-known fact, important e.g. for the classification of Hurwitz curves where $\Delta$ is maximal of type $(2,3,7)$.

Corollary 11 Different normal surface subgroups of a maximal triangle group belong to non-isomorphic quasiplatonic Riemann surfaces.

We should emphasize that the hypothesis that both surface groups are normally included in $\Delta$ is very important. If not, the situation can get much more complicated:

Example 1 The group $\Delta(2,3,2 n)$ contains (see Theorem 6 ) exactly three different copies $\Delta_{1}(2, n, 2 n), \Delta_{2}(2, n, 2 n), \Delta_{3}(2, n, 2 n)$ of a group of type $(2, n, 2 n)$, the intersection of them being $\Delta(n, n, n)$. The genus $g(g \geq 2)$ surface $X$ with affine equation

$$
y^{2}=x^{2 g+1}-1
$$

is uniformized by a normal subgroup $K$ of index $n$ in $\Delta(n, n, n)$ for $n=2 g+1$. It can be seen that $K \triangleleft \Delta_{1}(2, n, 2 n)$, but the inclusion of $K$ in the other two 
groups of type $(2, n, 2 n)$ is not normal, otherwise $K$ would be normal also in the maximal triangle group $\Delta(2,3,2 n)$. This is impossible since Aut $K$ is not of order $6 n$ but only the direct product of a cyclic group $C_{n}$ acting on the $x$-plane with the factor $C_{2}$ coming from the hyperelliptic involution.

Since $\Delta_{2}$ and $\Delta_{3}$ are conjugate to $\Delta_{1}$ in $\Delta(2,3,2 n)$, we have conjugates $K_{2}, K_{3}$ to $K$ inside $\Delta(n, n, n)<\Delta_{1}$, but they are non-normal subgrups of $\Delta_{1}$, and the conjugating elements are not contained in $N\left(\Delta_{1}\right)=\Delta_{1}=N(\tilde{\Delta}), \tilde{\Delta}:=N(K)$.

\section{Multiple regular dessins of given type}

As already mentioned at the end of section 4.3, given a regular dessin $\mathcal{D}$ induced on the surface uniformized by $K$ by the inclusion $K \triangleleft \Delta$, the number of regular dessins on $X$ of the same type as $\mathcal{D}$ is bounded by $n_{\tilde{\Delta}}(\Delta)$, where $\tilde{\Delta}=N(K)$. As indicated, we will count dessins on $X$ only as "different" if they are induced by different triangle groups $\Delta \neq \alpha \Delta \alpha^{-1}$, i.e. do not result from each other by renormalization. The number of all dessins of the same type as $\mathcal{D}$ can differ from $n_{\tilde{\Delta}}(\Delta)$ by two reasons. On the one hand, $K$ may be contained as a nonnormal subgroup in triangle groups conjugate to $\Delta$, see Example 1 above. On the other hand, even if it seems to be hard to give a concrete counterexample, it is not at all clear if $K$ is necessarily contained in all conjugate triangle groups $\alpha^{-1} \Delta \alpha, \alpha \in C(\tilde{\Delta}, \Delta)$.

Using Section 5 we can give now more precise information on multiple quasiplatonic surfaces with several regular dessins of the same type.

Theorem 12 Suppose that $\mathcal{D}_{1}$ and $\mathcal{D}_{2}$ are two regular dessins of the same type, but not of type $(n, 2 n, 2 n), n>2$, on a quasiplatonic surface $X$. Then there is an automorphism of $X$ sending $\mathcal{D}_{1}$ to $\mathcal{D}_{2}$.

Proof.- Suppose that $X$ is uniformized by a Fuchsian group $K$. The dessins $\mathcal{D}_{j}, j=1,2$, are determined by the normal inclusion of $K$ inside two triangle groups $\Delta_{1}$ and $\alpha \Delta_{1} \alpha^{-1}$, both contained in the normalizer $\tilde{\Delta}=N(K)$, which is necessarily another triangle group. Now we know $\alpha \in C\left(\tilde{\Delta}, \Delta_{1}\right)=N(\tilde{\Delta}) N\left(\Delta_{1}\right)$ and we may even omit the factor $N\left(\Delta_{1}\right)$ - its effect is only renormalization of the dessin. If $\alpha \in \tilde{\Delta}$, it represents an automorphism of $X$, so the claim is true with possible exceptions in cases where

- $\tilde{\Delta}$ is strictly contained in $N(\tilde{\Delta})$,

- and $N\left(\Delta_{1}\right)$ does not contain $N(\tilde{\Delta})$.

The second condition implies in particular that $\Delta_{1}$ is strictly contained in $\tilde{\Delta}$. Therefore we have to consider only the two-step inclusions $\Delta_{1}<\tilde{\Delta}<N(\tilde{\Delta})$ discussed in Section 5. Taking into account $N(\tilde{\Delta}) \nsubseteq N\left(\Delta_{1}\right)$, a look over these possible inclusion relations shows that the obstruction in question can only occur for

$$
\Delta_{1}=\Delta(n, 2 n, 2 n), \tilde{\Delta}=\Delta(2,2 n, 2 n), N(\tilde{\Delta})=\Delta(2,4,2 n)
$$


the exceptional types mentioned in the theorem.

Example 2 The lowest genus example of such an exceptional multiple dessin lives on the genus 4 quasiplatonic curve $X=\mathbb{D} / K$ given by the affine equation

$$
y^{3}=1-x^{6} .
$$

Its covering group $K$ is a normal subgroup of $\Delta_{1}=\Delta(3,6,6)$ with quotient $\Delta_{1} / K \cong C_{3} \times C_{6}$, but $\tilde{\Delta}=N(K)=N\left(\Delta_{1}\right)=\Delta(2,6,6)$ with quotient Aut $X=\tilde{\Delta} / K \cong S_{3} \times C_{6}$. On the other hand

$$
N(\tilde{\Delta})=\Delta(2,4,6)
$$

contains $\tilde{\Delta}$ with index 2 , so the action of $N(\tilde{\Delta})$ induces two different regular dessins of type $(3,6,6)$ on $X$. In fact, $\Delta(2,6,6)$ contains two normal subgroups $\Delta_{1}, \Delta_{2}$ both of type $3,6,6$ and both containig $K$ as a normal subgroup what can be seen as follows. If we denote the elements of $C_{6}$ by representants of the residue classes $\mathbb{Z} / 6 \mathbb{Z}$ we can give the canonical homomorphism of $\Delta(2,6,6)$ onto Aut $X=\tilde{\Delta} / K \cong S_{3} \times C_{6}$ by defining the images of the generators

$$
\gamma_{0} \mapsto((23), 3), \quad \gamma_{1} \mapsto((12), 2), \quad \gamma_{\infty} \mapsto((123), 1) .
$$

The generator triples of $\Delta_{1}$ and $\Delta_{2}$ are

$$
\gamma_{1}^{2}, \gamma_{0} \gamma_{\infty} \gamma_{0}, \gamma_{\infty} \text { and } \gamma_{\infty}^{2}, \gamma_{0} \gamma_{1} \gamma_{0}, \gamma_{1},
$$

respectively. It is easy to see that the canonical homomorphism maps $\Delta_{1}$ onto $C_{3} \times C_{6}$ and $\Delta_{2}$ onto $S_{3} \times C_{3}$.

Geometrically we have the following picture, easily understandable after a look on the inclusion of $\Delta_{1}$ in $\tilde{\Delta}$ and $N(\tilde{\Delta})$ (see Figure 5, where the tesselations for $\Delta_{1}, \tilde{\Delta}$ and $N(\Delta)$ are depicted with dashing black lines, solid grey lines and thick white lines respectively). If we draw $\mathcal{D}_{1}$ such that the $\Delta_{1}$-fixed points of order 6 become the vertices of $\mathcal{D}_{1}$ on $X$, the conjugation by the order 2 generator of $\tilde{\Delta}$ interchanges only the colour of the vertices, but the new order 2 generator of $N(\tilde{\Delta})$ interchanges vertices and centers of faces, so the underlying graph of $\mathcal{D}_{2}$ is dual to the graph of $\mathcal{D}_{1}$ (and its vertices can be coloured in two ways). The same happens for all exceptional multiple dessins excluded in Theorem 9 , so we obtain finally

Theorem 13 Two regular dessins $\mathcal{D}_{1}, \mathcal{D}_{2}$ of the same type on a quasiplatonic Riemann surface $X$ of genus $g>1$ can be transformed into each other by

1. renormalization, i.e. permuting $0,1, \infty$ in the associate Belyi functions,

2. automorphisms of $X$ or

3. passing to the dual graph (in some cases of type $(n, 2 n, 2 n)$ only).

Only in the third case it can happen that the automorphism groups of $\mathcal{D}_{1}$ and $\mathcal{D}_{2}$ are not isomorphic, see Example 2 . 


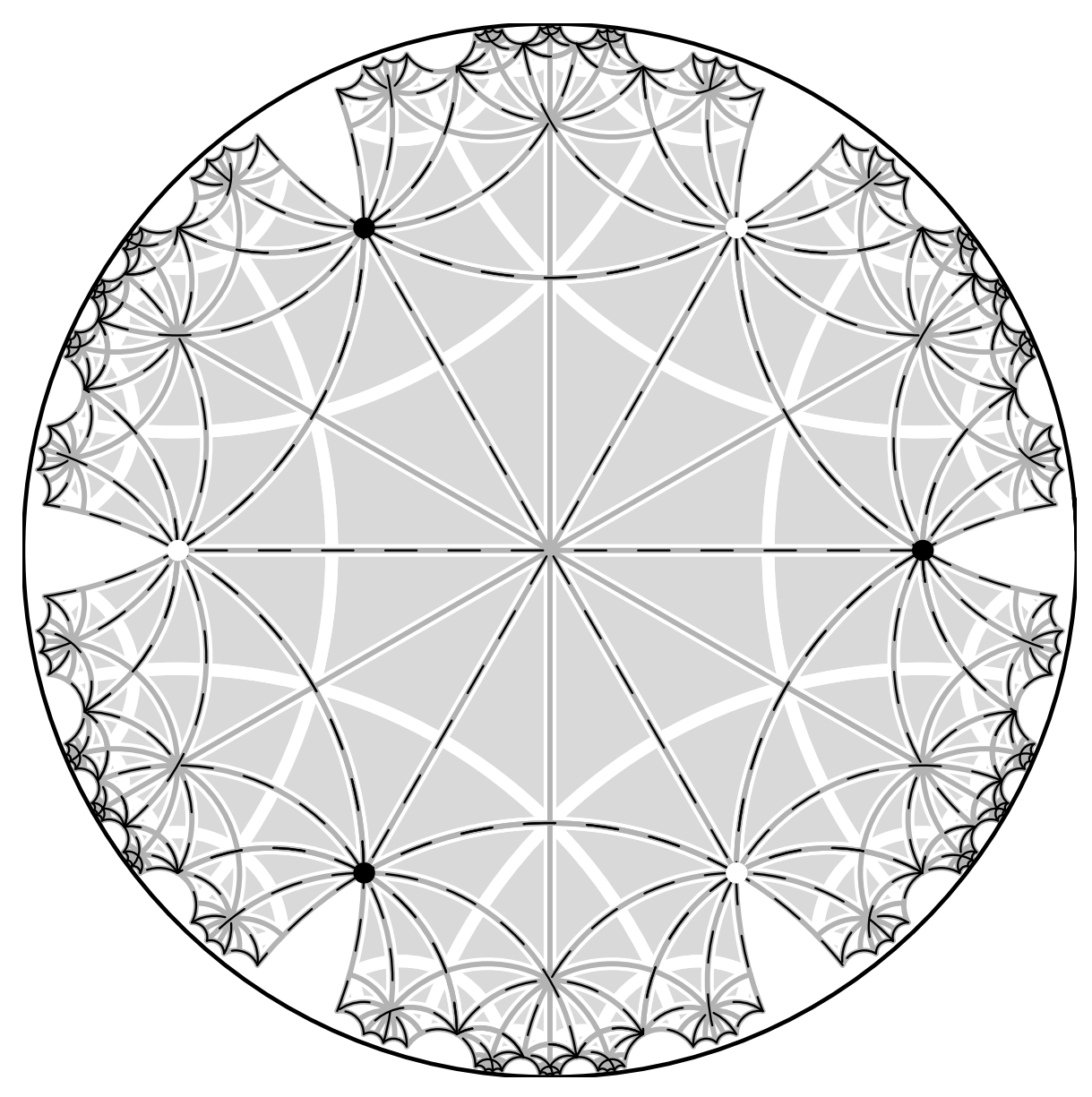

Figure 5: Inclusions related to the exceptional case $n=3$. 


\section{References}

[1] P. B. Cohen, C. Itzykson, J. Wolfart, Fuchsian triangle groups and Grothendieck dessins. Variations on a theme of Belyi, Comm. Math. Physics 163 (1994), 605-627.

[2] E. Girondo, Multiply quasiplatonic Riemann surfaces, Experimental Mathematics 12 (4) (2003), 463-475.

[3] E. Girondo, Homepage. http://www.uam.es/ernesto.girondo

[4] G.A. Jones and D. Singerman: Belyi functions, hypermaps and Galois groups, Bull. London Math. Soc. 28 (1996), 561-590.

[5] G. Margulis, Discrete subgroups of semisimple Lie groups. Springer-Verlag 1991.

[6] T. Miyake, Modular Forms. Springer-Verlag 1989.

[7] D. Singerman, Finitely Maximal Fuchsian Groups, J. London Math. Soc. (2) 6 (1972), 29-38.

[8] D. Singerman, R Syddall, The Riemann Surface of a Uniform Dessin, Contributions to Algebra and Geometry 44 (2003), 413-430.

[9] M. Streit, Field of definition and Galois orbits for the Macbeath-Hurwitz groups, Arch. Math. 74 (2000), 342-349.

[10] M. Streit and J. Wolfart, Characters and Galois invariants of regular dessins, Revista Matématica Complutense 13 (2000), 49-81.

[11] K. Takeuchi, Arithmetic triangle groups, J. Math. Soc. Japan, 29 (1977), 91-106.

[12] K. Takeuchi, Commensurability classes of arithmetic triangle groups, J. Fac. Sci. Univ. Tokio Sect. 1A Math. (1) 24 (1977), 201-212.

[13] J. Wolfart, The "obvious" part of Belyi's theorem and Riemann surfaces with many automorphisms, in L. Schneps and P. Lochak (eds.) Geometric Galois actions 1, 97-112, L.M.S. Lecture Notes Series 242 (1997), Cambridge U. Press.

[14] J. Wolfart: Triangle groups and Jacobians of CM type, manuscript at http://www.math.uni-frankfurt.de/ ${ }^{\sim}$ wolfart/.

Ernesto Girondo

Departamento de Matemt́icas, Universidad Autónoma de Madrid 28049 Madrid (Spain)

Jürgen Wolfart

Fachbereich Mathematik, Johann Wolfgang Goethe-Universität Robert-Mayer-Str. 6-8, 60054 Frankfurt am Main (Germany) 